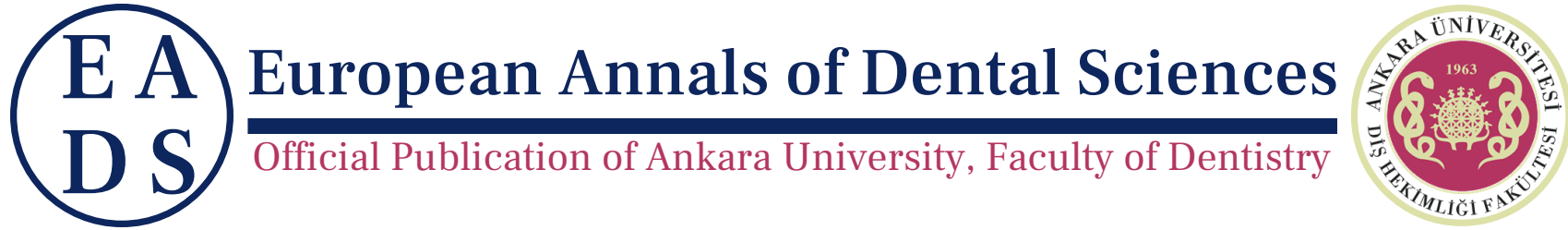

EADS, 2021, $48(2), 52-56$

\title{
The Internal and Marginal Adaptation of Zirconia Based Fixed Dental Prostheses in the Posterior Region
}

\author{
Ayşe Koçak-Büyükdere ${ }^{1, *}$ and Atilla Sertgöz $\odot 2$ \\ ${ }^{1}$ DDS, PhD, Kocaeli University, Faculty of Dentistry, Department of Prosthetic Dentistry, Kocaeli, TURKEY \\ and ${ }^{2} \mathrm{DDS}, \mathrm{PhD}$, Private Clinic, Istanbul, TURKEY \\ *Corresponding Author; akocakbuyukdere@gmail.com
}

\begin{abstract}
Purpose: Long-term clinical success is closely related to the internal and marginal adaptation of the restorations. The purpose of this study was to evaluate the internal adaptation of zirconia-based fixed dental prostheses in the posterior region.

Materials \& Methods: Four-unit porcelain-fused to zirconia fixed dental prostheses were fabricated with CAD-CAM system in 20 patients. For the evaluation of the restorations' internal fit, conventional silicone replicas were used with the optical microscope. The obtained silicone impression was cut to obtain two cross-sections from buccolingual and 3 from mesiodistal. Fifty different measurements were taken from every tooth by taking 10 from each cross- section; 2 from the margin, 6 from the axial surfaces and 2 from the occlusal by the optical microscope.

Results: There were statistically significant differences in internal discrepancy in terms of area and tooth type. The average internal discrepancy on the axial surfaces of the molars was $142.39 \pm 47.42 \mu \mathrm{m}$. In premolars, the mean was $139.53 \pm 46.80 \mu \mathrm{m}$. The average of the internal discrepancy values obtained from occlusal surfaces of the molar teeth was $164.39 \pm 53.61 \mu \mathrm{m}$, whereas the same average for the premolar teeth was $161.92 \pm 60.54 \mu \mathrm{m}$.

Conclusion: Within the limitations of this study, restorations fabricated by CAD/CAM and internal and marginal discrepancies were at clinically acceptable intervals.
\end{abstract}

Key words: CAD/CAM; internal discrepancy; marginal discrepancy

\section{Introduction}

Development of computer-aided design (CAD)/computeraided manufacturing (CAM) technology, various dental restorations are beginning to be formulated digitally. The presence of technicians continues in CAD/CAM technology, is preferred to process different porcelains on a substructure to obtain more esthetic results. ${ }^{1}$

Cement dissolution is one of the most critical factors causing secondary caries. The sensitivity of the restorations at the laboratory stage affects the cement thickness due to its internal and marginal fit. ${ }^{2,3}$ The success of all restoration types used in dentistry is highly related to the marginal fit of the restorations, and it has been reported that a 50-200 $\mu \mathrm{m}$ marginal gap is clinically acceptable. ${ }^{2-9}$

The factors affecting the internal and marginal adaptations indicate that fit of the selected restoration with the tooth struc- ture and wear resistance is effective. ${ }^{10}$ Another factor is the location of the teeth, which determines the tooth preparation. It has been reported that restorations in the mandibular posterior region require more tooth preparation. Although it is recommended to provide at least 2-6 degrees of taper, it has been stated that the desired durability is maintained at 6-12 degrees. ${ }^{11-14}$ Depending on the impression material and the precision of the impression technique, better fit will be achieved in restorations based on obtained models. ${ }^{15}$ In CAD/CAM systems, however, a higher number of measured points which are transferred to the system, will result in a more precise restoration plan. ${ }^{16}$

Various techniques have been used to determine marginal and internal gaps as follows; direct view, cross-sectional technique after cementation and embedding, impression technique, weighing the light-body additional silicone, and explorer com- 


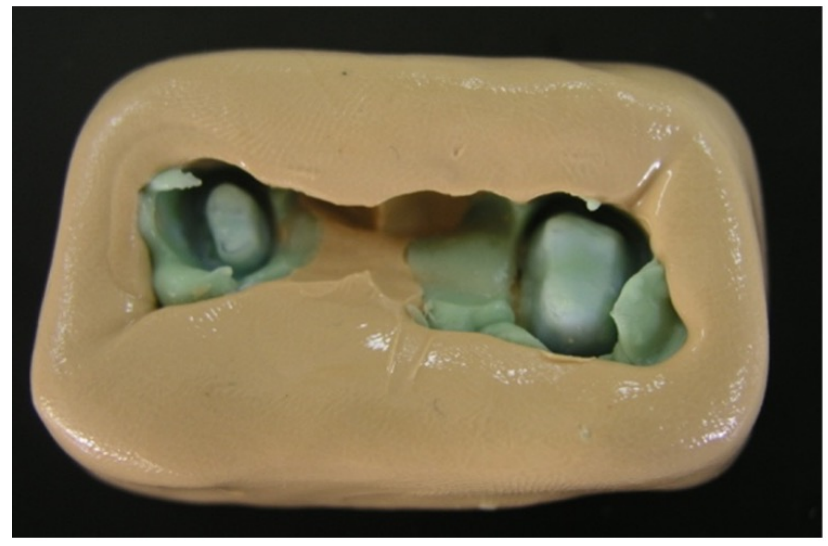

Figure 1. Silicone model which were obtained before cementation

bined with visual examination. Marginal discrepancies can be detected on the model by microscopic examination. Another method is to section the samples after cementation and examine their inner surfaces under a microscope or micro-CT can be used as a non-sectional method. ${ }^{1,8,17-19}$

The purpose of this study was to determine the internal and marginal gaps of 4-unit zirconia-based fixed partial dentures that were applied to the posterior region by using an impression technique (replica method) and non-invasive method. The null hypothesis of the study was that the internal and marginal gaps would be within clinical limits in both teeth type, and also gaps in the margin, axial and occlusion would be similar.

\section{Materials and Methods}

This study was conducted upon the approval of the Marmara University Health Science Department Ethics Board under protocol number MAR-YÇ-2005-0082. Tooth preparations involved chamfer finish lines. The restoration process included impression taking, coping design by dental technician, coping fabrication with CAD/CAM technology, and porcelain layering by dental technician. Four-unit zirconia-based fixed partial dentures (CERCON Smart, DeguDent GmbH, Germany) were fabricated. To determine the marginal and internal fit of the mentioned fixed partial dentures, impression were taken from the inner surfaces of the restorations, and the cement gaps were identified under an optical microscope. To check the internal discrepancy of the zirconia-based fixed partial dentures, all extraneous material of the temporary fixed partial dentures were removed before the cementation stage. The surfaces of the teeth were cleaned by brushs. An addition-type thin consistency silicone material (Affinis, Coltène, Switzerland) was applied on the prepared teeth. After the restorations were placed over the teeth, the patient was asked to bite. After the impression material had set, ${ }^{2,8,9}$ the restoration was removed. An addition-type medium-consistency silicone impression material (Affinis, Coltène, Switzerland) was applied to internal surface of the restoration over the thin-consistency impression material left inside the restoration to support the thinner layer. After the medium-consistency silicone material was set, an addition-type thick-consistency silicone (Affinis, Coltène, Switzerland) was applied in order to help remove the replica from the restoration in one piece (Figure 1).

The set silicone impression was crosscut to obtain two buccolingual and three mesiodistal cross-sections (Figure 2). Fifty different cement thickness measurements were taken from each tooth by taking 10 measurements from each cross-section; 2 from the margin, 6 from the axial surface and 2 from the

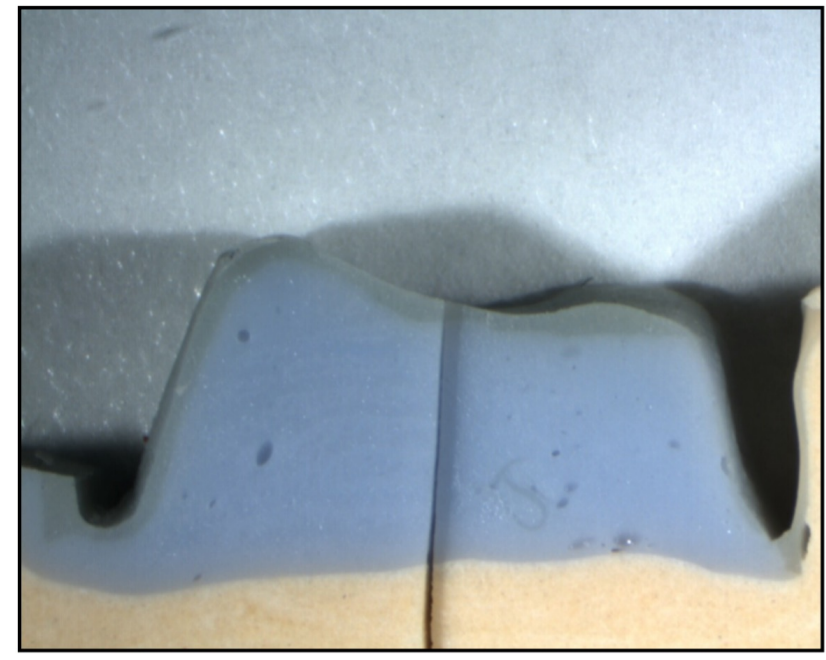

Figure 2. Internal measurements were shown in details, a: margin, b: axial, c: occlusion

occlusal surface with an optical microscope with a $3.2 \mathrm{x}$ optical scanner, $48 \mathrm{x}$ microscopic magnification, and $102.81 \mathrm{x}$ screen magnification. Average occlusal, proximal, and marginal adaptation values were obtained by calculating the mean of the three times measurements in the internal silicone replicas.

\section{Statistical Analyses}

For evaluating the findings obtained during the study, a commercially available software (IBM SPSS Statistics 22 IBM, Turkey) was used for statistical analyses. Data was tested for normality using Kolmogorov-Smirnov (Shapiro Wilks) test, and was found to be normally distributed. Two-way ANOVA was used to identify the effect of the marginal gap and the tooth type $(\alpha=0.05)$. One-way ANOVA (post hoc Tamhane's T2 test) and Student $t$ test were used as attendance tests. Significance was evaluated at the $\alpha=0.05$ level.

\section{Results}

There was a significant difference between the tooth type and internal adaptation $(\mathrm{p}=0.003 ; \mathrm{p}<0.05)$. There was not a significant difference between the location of the gaps and the internal adaptation $(\mathrm{p}=0.113 ; \mathrm{p}>0.05)$. There was a significant difference between the tooth type and gap regions $(p=0.000$; $\mathrm{p}<0.05$ ). Teeth type and gap regions had an effect on the internal adaptation values (Table 1 ).

Internal adaptations of the molar teeth were larger than premolar teeth and were statistically significantly higher than the premolar teeth $(\mathrm{p}=0.010 ; \mathrm{p}<0.05)$ (Table 2$)$. There was not a statistically significant difference between the axial gaps of the molar and premolar teeth $(\mathrm{p}=0.198 ; \mathrm{p}>0.05)$. There was $\mathrm{a}$ statistically significant difference between the occlusal gaps of the molar and premolar teeth $(\mathrm{p}=0.000 ; \mathrm{p}<0.05)$. There was a statistically significant difference between the marginal gaps of the molar and premolar teeth $(\mathrm{p}=0.000 ; \mathrm{p}<0.05)$. Marginal gaps of the premolar teeth were larger than the marginal gaps of the molar teeth. There was a statistically significant difference between the gap regions and the molar teeth $(\mathrm{p}=0.000 ; \mathrm{p}<0.05)$. Post hoc Tamhane's T2 test indicated that occlusal gaps were larger than the axial and the marginal gaps ( $1=0.000 ; \mathrm{p} 2=0.000 ; \mathrm{p}<0.05)$. Axial gaps were significantly larger than the marginal gaps $(\mathrm{p} 1=0.000 ; \mathrm{p}<0.05)$. There was a statistically significant difference between the gap regions 
Table 1. Internal adaptation of tooth type and gap regions by 2-Way ANOVA test.

\begin{tabular}{ccccc}
\hline Source & Type III Sum of Squares & df & Mean Square & F \\
\hline Tooth type & 21511.76 & 1 & 21511.76 & 9.123 \\
Gap regions & 10284.99 & 2 & 5142.492 & 2.181 \\
Tooth * Gap regions & 687778 & 2 & 343889 & 145.839 \\
\hline
\end{tabular}

$* \mathrm{p}<0.05$

Table 2. Mean internal gap widths according to tooth type and region

\begin{tabular}{|c|c|c|c|c|}
\hline & & \multicolumn{2}{|c|}{ Internal Adaptation } & \multirow[b]{2}{*}{$\mathrm{p}$} \\
\hline & & $\mathrm{n}$ & Mean $_{ \pm} \mathrm{SD}$ & \\
\hline \multirow[t]{2}{*}{ Tooth type } & Molar & 1500 & $x_{142.70 \pm 50.59}$ & ${ }^{1} 0.010^{*}$ \\
\hline & Premolar & 1500 & $\mathrm{y}_{137.90 \pm 51.15}$ & \\
\hline \multirow[t]{3}{*}{ Region } & Axial & 1800 & ${ }^{a} 140.97 \pm 47.12$ & ${ }^{2} 0.138$ \\
\hline & Occlusion & 600 & $\mathrm{a}_{136.67 \pm 54.05}$ & \\
\hline & Margin & 600 & ${ }^{a} 141.92 \pm 58.07$ & \\
\hline
\end{tabular}

${ }^{1}$ Student $\mathrm{t}$ test ${ }^{2}$ Oneway ANOVA Test $* \mathrm{p}<0.05$

$\mathrm{x}-\mathrm{y}$ show the differences between the molar and premolar

a show the both teeth's measurements.

Table 3. Mean gap widths according to the region in tooth

\begin{tabular}{|c|c|c|c|c|c|}
\hline \multirow[b]{3}{*}{ Gap region } & \multicolumn{4}{|c|}{ Tooth Type } & \multirow[b]{3}{*}{${ }^{1} \mathrm{p}$} \\
\hline & \multicolumn{2}{|c|}{ Molar } & \multicolumn{2}{|c|}{ Premolar } & \\
\hline & $\mathrm{n}$ & Mean $_{ \pm} \mathrm{SD}$ & $\mathrm{n}$ & Mean $_{ \pm} \mathrm{SD}$ & \\
\hline Axial & 900 & $\mathrm{ax}_{142.40 \pm 47.43}$ & 900 & $\mathrm{ax}_{139.54 \pm 46.8}$ & 0.198 \\
\hline Occlusion & 300 & $\mathrm{bx}_{164.39 \pm 53.62}$ & 300 & by $_{108.94 \pm 37.89}$ & $0.000 *$ \\
\hline Margin & 300 & $\mathrm{cx}_{121.91 \pm 47.81}$ & 300 & ${ }^{c y} 161.93 \pm 60.55$ & $0.000 *$ \\
\hline${ }^{2} \mathrm{p}$ & & $0.000^{*}$ & & $0.000^{*}$ & \\
\hline
\end{tabular}

${ }^{1}$ Student $\mathrm{t}$ test ${ }^{2}$ Oneway ANOVA Test $* \mathrm{p}<0.05$

a,b,c show the region differences

$x-y$ show the differences of the tooth type

and the premolar teeth $(\mathrm{p}=0.000 ; \mathrm{p}<0.05)$. Post hoc Tamhane's $\mathrm{T} 2$ test indicated that marginal gaps were larger than the axial and the occlusal gaps ( $1=0.000 ; \mathrm{p} 2=0.000 ; \mathrm{p}<0.05)$. Axial gaps were significantly larger than the occlusal gaps $(\mathrm{p} 1=0.000$; $\mathrm{p}<0.05$ ) (Table 3).

\section{Discussion}

The null hypothesis of the study was the internal and marginal gap would be within clinical limits was accepted but the other null hypothesis about the gaps in the margin, axial and occlusion would be similar was rejected. It has been reported that the involvement of multiple steps and technique sensitivity of laboratory procedures can affect marginal adaptation and internal fit, which is clinically essential for the longevity of a restoration. ${ }^{8,18}$ In this study, impression technique (replica method) was used for evaluating marginal and internal fit, which is considered a reliable and non-invasive method for measuring fit of the restoration. In this technique, the cement is replaced with the impression material, and the restoration is placed on the abutment tooth. The restoration and impression material are separated from the abutment, and the thickness of the cement layer analogue is measured. However, the impression replica technique has certain limitations such as tearing of the elastomeric film upon removal from the crown and errors in sectioning which would eventually lead to overestimated measurements. Too much marginal gap could lead to increased cement thickness, secondary caries risk, and pulpal irritation. ${ }^{20,21}$ On the other hand, incorrect fit of the internal adaptation may cause axial walls and occlusal surfaces to not be in ideal contact with the restoration, thereby reducing the retention and leading to restoration farcture. ${ }^{22} 22$ Before the cementation of the restorations in our study, we identified internal and marginal discrepancies with the help of measurements on sectioned silicone. The number of measurements made in the internal adaptation studies were significant. Only accurate number of the section could inform on the internal structure of the entire restoration be obtained. Therefore, the number of sections should be increased as much as possible. ${ }^{23}$ In our study, we took a total of 50 measurements from each tooth.

It is possible to perform standard crosscuts for internal fit evaluations and be ensured that the operator can observe every surface in the best way when cutting in vitro; whereas it is not possible to achieve outcome in vivo. ${ }^{1}$ It has been reported that 50-200 $\mu \mathrm{m}$ marginal gap width is clinically acceptable. ${ }^{2,3,5-9}$ When the total internal fit was examined in our study, an average of $139.16 \pm 49.01 \mu \mathrm{m}$ was found, which was within the clinically acceptable range.

In a study using a different CAD/CAM system, it was observed that gap width was increased from the marginal edge to the occlusal surface. ${ }^{19,22,24,25}$ It was reported that abovementioned result was due to the intraoral scanner being unable to detect some curved surfaces because of the length of the scanner tip's diameter. In our study, the highest internal discrepancy values $(164.39 \pm 53.62 \mu \mathrm{m})$ were measured on the molar occlusal surface, and the lowest values (108.94 \pm 37.89 $\mu \mathrm{m}$ ) were measured on the premolar occlusal surface. Inclined surfaces on the molar teeth occlusions were affected the abil- 
ity of the scanner to scan the crown or fixed partial dentures perfectly in the CAD/CAM.

If the scanner is able to detect the surfaces clearly, gaps would be lower than $100 \mu \mathrm{m} .{ }^{5,26}$ Boening et al. reported an average marginal gap width of $80-95 \mu \mathrm{m}$ in anterior teeth and $90-145 \mu \mathrm{m}$ in posterior teeth. It has been stated that the Procera AllCeram system showed clinically acceptable values. ${ }^{2}$ In our study, when the molar teeth and premolar teeth were compared, the sum of the internal discrepancy values for premolar teeth was $137.90 \pm 51.15 \mu \mathrm{m}$, whereas it was $142.70 \pm 50.59 \mu \mathrm{m}$ for molar teeth. It could be said that the internal discrepancy of the molar teeth was higher since the preparation of the molar teeth is more complicated than that of the premolar teeth; in particular, the distal part was not clear, and the marginal region was harder to scan.

In the study by de Paula Silveira et al. ${ }^{4}$ the mean axial integrity value was $138 \mu \mathrm{m}$ in different restorations, and a similar result was reported by Tabata et al. ${ }^{1}$ In our study, the mean axial integrity value was $140.97 \pm 47,12 \mu \mathrm{m}$ in ceramics, similar to the Paula Silveira et al and Tabata et al results. Tabata et al. also reported that ceramic restorations have a maximum integrity of $232 \pm 29 \mu \mathrm{m}$, and in this study, the occlusal integrity was $164,39 \pm 53,62 \mu \mathrm{m}$, which was the largest of all gaps.

\section{Conclusion}

As a result of the internal adaptation evaluation of zirconiabased fixed partial dentures, the broadest gap was measured in the occlusal surface and the narrowest was measured in the margins. When the abutment teeth were examined, internal and marginal discrepancies were found in the molar teeth compared to the premolar teeth. Internal and marginal discrepancies in zirconia-based fixed partial dentures prepared by CAD/CAM technology were acceptable for clinical service.

\section{Author Contributions}

Research concept and design: A.K.B, A.S. Collection and/or assembly of data: A.K.B. Data analysis and interpretation: A.K.B, A.S. Writing the article: A.K.B, A.S. Critical revision of the article: A.K.B. Final approval of article: A.K.B.

\section{Conflict of Interest}

Authors declare that have no conflict of interests.

\section{Authors' ORCID(s)}

A.K.B. $\quad 0000-0003-1942-456 \mathrm{X}$

A.S. $\quad 0000-0002-3018-4467$

\section{References}

1. Tabata LF, de Lima Silva TA, de Paula Silveira AC, Ribeiro APD. Marginal and internal fit of CAD-CAM composite resin and ceramic crowns before and after internal adjustment. J Prosthet Dent. 2020;123(3):500-505. doi:10.1016/j.prosdent.2019.01.010.

2. Boening KW, Wolf $\mathrm{BH}$, Schmidt $\mathrm{AE}$, Kästner K, Walter $\mathrm{MH}$. Clinical fit of Procera AllCeram crowns. J Prosthet Dent. 2000;84(4):419-24. doi:10.1067/mpr.2000.109125.

3. Papadiochou S, Pissiotis AL. Marginal adaptation and CAD-CAM technology: A systematic review of restora- tive material and fabrication techniques. J Prosthet Dent. 2018;119(4):545-551. doi:10.1016/j.prosdent.2017.07.001.

4. de Paula Silveira AC, Chaves SB, Hilgert LA, Ribeiro AP. Marginal and internal fit of CAD-CAM-fabricated composite resin and ceramic crowns scanned by 2 intraoral cameras. J Prosthet Dent. 2017;117(3):386-392. doi:10.1016/j.prosdent.2016.07.017.

5. May KB, Russell MM, Razzoog ME, Lang BR. Precision of fit: the Procera AllCeram crown. J Prosthet Dent. 1998;80(4):394-404. doi:10.1016/s0022-3913(98)700022.

6. Oz FD, Bolay S. Comparative Evaluation of Marginal Adaptation and Fracture Strength of Different Ceramic Inlays Produced by CEREC Omnicam and Heat-Pressed Technique. Int J Dent. 2018;2018:5152703. doi:10.1155/2018/5152703.

7. Padrós R, Giner L, Herrero-Climent $M$, Falcao-Costa $C$, Ríos-Santos JV, Gil FJ. Influence of the CAD-CAM Systems on the Marginal Accuracy and Mechanical Properties of Dental Restorations. Int J Environ Res Public Health. 2020;17(12). doi:10.3390/ijerph17124276.

8. Sorensen JA. A standardized method for determination of crown margin fidelity. J Prosthet Dent. 1990;64(1):18-24. doi:10.1016/0022-3913(90)90147-5.

9. Tinschert J, Natt G, Mautsch W, Spiekermann H, Anusavice KJ. Marginal fit of alumina-and zirconia-based fixed partial dentures produced by a CAD/CAM system. Oper Dent. 2001;26(4):367-74.

10. Nissan J, Rosner O, Rosen G, Naishlos S, Zenziper E, Zelikman $\mathrm{H}$, et al. Influence of Vinyl Polysiloxane Impression Techniques on Marginal Fit of Metal Frameworks for Fixed Partial Dentures. Materials (Basel). 2020;13(20). doi:10.3390/ma13204684.

11. Al Mortadi N, Bataineh $\mathrm{K}$, Al Janaideh $\mathrm{M}$. Fatigue Failure Load of Molars with Thin-Walled Prosthetic Crowns Made of Various Materials: A 3D-FEA Theoretical Study. Clin Cosmet Investig Dent. 2020;12:581-593. doi:10.2147/ccide.S286826.

12. Malone W, Koth D. Tylman's Theory and Practice of Fixed Prosthodontics. 8 [sup] th ed. Missouri: Ishiyaku Euro America. 1989:237-54.

13. Rosenstiel SF, Land MF. Contemporary fixed prosthodontics-e-book. Elsevier Health Sciences; 2015.

14. Shillingburg HT, Hobo S, Whitsett LD, Jacobi R, Brackett S. Fundamentals of fixed prosthodontics. vol. 194. Quintessence Publishing Company; 1997.

15. McCabe JF, Walls AW. Applied dental materials. John Wiley \& Sons; 2013.

16. Duret F, Blouin JL, Duret B. CAD-CAM in dentistry. J Am Dent Assoc. 1988;117(6):715-20. doi:10.14219/jada.archive.1988.0096.

17. Abduo J, Lyons K, Swain M. Fit of zirconia fixed partial denture: a systematic review. J Oral Rehabil. 2010;37(11):86676. doi:10.1111/j.1365-2842.2010.02113.x.

18. $\mathrm{Ng} \mathrm{J}$, Ruse D, Wyatt C. A comparison of the marginal fit of crowns fabricated with digital and conventional methods. J Prosthet Dent. 2014;112(3):555-60. doi:10.1016/j.prosdent.2013.12.002.

19. Peng CC, Chung KH, Yau HT, Ramos J V. Assessment of the internal fit and marginal integrity of interim crowns made by different manufacturing methods. J Prosthet Dent. 2020;123(3):514-522. doi:10.1016/j.prosdent.2019.02.024.

20. Irie M, Suzuki K, Watts DC. Marginal and flexural integrity of three classes of luting cement, with early finishing and water storage. Dent Mater. 2004;20(1):3-11. doi:10.1016/s0109-5641(03)00052-6.

21. Lindquist TJ, Connolly J. In vitro microleakage of luting cements and crown foundation material. J Prosthet Dent. 2001;85(3):292-8. doi:10.1067/mpr.2001.113705. 
22. Reich S, Wichmann M, Nkenke E, Proeschel P. Clinical fit of all-ceramic three-unit fixed partial dentures, generated with three different CAD/CAM systems. Eur J Oral Sci. 2005;113(2):174-9. doi:10.1111/j.1600-0722.2004.00197.x.

23. Aktepe E. CAD-CAM Cerec 3 Sistemiyle Hazırlanan İnleylerin Marjinal Adaptasyonlarının İn Vitro Olarak Değerlendirilmesi Marmara Üniversitesi [Thesis]; 2005.

24. Bindl A, Mörmann WH. Marginal and internal fit of all-ceramic CAD/CAM crown-copings on chamfer preparations. J Oral Rehabil. 2005;32(6):441-7. doi:10.1111/j.1365-
2842.2005.01446.X.

25. Kokubo Y, Nagayama Y, Tsumita M, Ohkubo C, Fukushima $\mathrm{S}$, Vult von Steyern P. Clinical marginal and internal gaps of In-Ceram crowns fabricated using the GN-I system. J Oral Rehabil. 2005;32(10):753-8. doi:10.1111/j.13652842.2005.01506.x.

26. Nakamura T, Nonaka $M$, Maruyama $T$. In vitro fitting accuracy of copy-milled alumina cores and all-ceramic crowns. Int J Prosthodont. 2000;13(3):189-93. 